\title{
LECTURA Y ESCRITURA COMO OBJETO DE REFLEXIÓN E INTERVENCIÓN CONSTANTE EN EL AULA
}

* Magister en Educación. Instituto Tecnológico San Agustín Rectora. Kra 6 No 33 02. Ciudad: Monteria Correo: liana emb@ hotmail.com

**Estudiante de Español y literatura de la Universidad de Córdoba. C. 12 trans 15 No. 11-53 B. edmundolópez. Ciudad: montería. Correo: odsamael@ gmail.com

***Estudiante de Español y literatura de la Universidad de Córdoba Estudiante de Español y literatura de la Universidad de Córdoba. C.12 trans 15 No. $11-53$ B edmundolopez. Ciudhas: edmundolopez. Ciudad: giopova@gmail.com

\author{
READING AND WRITING AS AN OBJECT OF REFLECTION AND \\ CONSTANT INTERVENTION IN THE CLASSROOM
}

Recibido: 25 de febrero 2016 - Aprobado: 12 de julio de 2016

\section{RESUMEN}

En el presente artículo se estudiarán las distintas propuestas que han surgido en torno a la enseñanza de la lectura y la escritura en la escuela a partir de la revisión bibliográfica de teóricos iberoamericanos, entre 1993 y 2012 con el propósito de ir identificando el lugar de la lectura en el aula; analizar la forma en como se viene enseñando pese a las incontables dificultades que se enfrenta un docente en el ejercicio de su práctica. En esta revisión pudo notarse que como tendencia predominante entre los autores, se encuentra el enfoque socialconstructivista, orientado hacia la configuración, comprensión e integración de nuevos conceptos como producto de la interacción con el medio. Así mismo, se observó la necesidad de incluir a las TIC en la enseñanza de la lectura y escritura como dadas las condiciones actuales del entorno educativo.

Palabras clave: lectura, escritura, enfoques, enseñanza, aprendizaje.

\begin{abstract}
In this article we will study the different proposals that have emerged about how to teach reading and writing in the school, based on the bibliographical revision of Ibero-American theories, between years 1993 and 2012, with the purpose of starting to see what the place of reading in the classroom is; analyzing the way this skill is taught, even with the uncountable difficulties a teacher can face in his praxis. In this review, we could observe the predominant trend existing among authors is the socio-constructivist approach, focused on the configuration, comprehension and integration of new concepts; as a result of the interaction with the environment. Likewise, it was observed the need to include ICT in the teaching of reading and writing as the current conditions in the teaching environment are created.
\end{abstract}

Keywords: reading, writing , approaches, teaching, learning. 


\section{Introducción}

Tr a lengua por ser un elemento social, vivo y sujeto a la temporalidad en la que está inmersa es cambiante. Este hecho ha generado una serie de $\_$paradigmas con relación a su enseñanza que merece atención perentoria, cuando de fortalecer y solidificar el ejercicio docente se trata. Por ello, este trabajo busca, por medio del análisis y la revisión de distintas propuestas teóricas, identificar no solo el enfoque predominante para la enseñanza de la lectura y la escritura, sino el lugar de la lectura y la escritura como objeto para la investigación y reflexión e intervención constante en el aula.

Pensar en la lectura y escritura, significa repensarse a sí mismo. Pues, es desde estos dos pilares del conocimiento, donde se hace efectivo el principio de toda formación integral y consciente. Tal es el rol docente en su manifestación más idealista. En área del lenguaje, son muchos los aspectos que repercuten a la hora de llevar a cabo "una buena práctica" que facilite la enseñanza y el aprendizaje de la lengua. ¿Cómo enseñar a leer?, ¿cómo potenciar la escritura?, ¿cómo mejorar la comprensión de lectura? son algunos de los interrogantes a los que, después de dos centurias de investigaciones y formulaciones de "proyectos" pedagógicos, difícilmente se resuelven, en la medida en que se han venido implementando estrategias e iniciativas que fomentan estos ejercicios.

Los avances teóricos en el campo de la pedagogía del lenguaje, con toda la gama temática que la circunscribe, ha sido prolífica en los últimos años, y el aporte iberoamericano no se ha relegado, hecho que, para el fundamento teórico de una nueva escuela del siglo XXI, puede en gran medida, resultar provechoso. Por tal motivo, a continuación se mencionarán a algunos de los autores que han aportado en torno al hecho de la lectura y la escritura como práctica fundamental y necesaria para la formación; Álvarez (1993) en su artículo titulado "Técnicas de lectura eficaz", expone diferentes métodos de lectura basados sobre los pilares de la rapidez, la comprensión y la memorización, los cuales contribuyen al alcance de una buena comprensión lectora. Según este autor, los estudiantes deben conseguir, en primera instancia, la facilidad lectora, etapa en la que se debe suprimir la vocalización, la labialización y la subvocalización, ya que son factores que hacen el proceso de lectura lento. En segundo lugar, conseguir una gran movilidad ocular además de ejercitar la extensión y precisión del campo visual, y por último, se considera indispensable que los estudiantes posean prerrequisitos de lectura o índices lingüísticos. Con estas técnicas, se cree que se desarrollarán 
tanto el gusto como las capacidades lectoras en los estudiantes, lo que daría como resultado menos fracaso escolar.

Así mismo, en su investigación Reyes Yolanda (2005) propone la implementación de la enseñanza de la lectura desde los primeros años de vida, a través del núcleo familiar y en el entorno social del niño. Aquí postula que la lectura de narraciones y cuentos estimula el interés por la lectura y promueve la adquisición de nuevos conocimientos en años posteriores. De esta manera, la autora busca que se implementen nuevas políticas en la primera infancia con base a la lectura y escritura, haciendo énfasis a la estimulación de saberes en los primeros años de vida.

No es un secreto que el entorno familiar es determinante a la hora de hablar de prácticas escriturales o lectoras, nótese el caso de Borges o de John Stuart Mill quienes antes de los ocho años, alcanzaron un nivel de lectura y escritura envidiables, gracias a la influencia directa de su entorno, este tipo de estímulos desde edades tempranas facilita la creación de "índices lingüísticos" que den acogida a la lectura y produzcan menos fracaso escolar en años posteriores.

Las escuelas son los primeros escenarios formales para el encuentro con la lectura y la escritura académica, es decir, con la lectura y escritura con propósito (que es lo que debería ser). No obstante, en la escuela actual, se está dejando de lado la posibilidad de vincular a los contenidos académicos con el entorno real del educando, "faltan estrategias pedagógicas, creativas y sistemáticas que propicien prácticas de lectura y escritura con sentido; es decir, que respondan a intereses y situaciones reales de comunicación de los estudiantes, acordes con su contexto social y cultural" (Doria \& Pacheco, 2012), lo que genera que los ejercicios de lectura y escritura queden suspendidos en una simple intención o pretensión del docente, "en un trabajo que adolece de un valor práctico para el estudiante" (Doria, 2000) hecho que debe ser replanteado. Leer implica comunicarse con las mentes más brillantes del pasado, como menciona Descartes en su Discurso del método, también es reconocimiento. Según Larrosa, la lectura es un escenario para la trasformación del yo y del mundo; el mundo está en la lectura. Por ello cuando se piensa en la lectura o la escritura como un instrumento más para evaluar se está vedando la posibilidad emancipadora del niño o del joven, se está transmitiendo la vejez conceptual y no se está dando paso a la lectura sobre el mundo que facilite la propia concepción del entorno. 
Sumado a lo anterior, Sánchez (1988), en su artículo titulado "Aprender a leer y leer para aprender: Características del escolar con pobre capacidad de comprensión" analiza la baja comprensión lectora, con el fin de describir las características de los sujetos que presentan dificultades para adquirir nuevos conocimientos a través de la lectura; Sánchez plantea que uno de los principales fracasos en la comprensión y aprendizaje por medio de la lectura es que los jóvenes aprenden a leer, pero no lo hacen leyendo, sino a través del descifre y reconocimiento de los símbolos propios de la escritura. Por lo anterior, la lectura y la escritura no son un proceso involuntario o automático, puesto que está inmerso en un entramado de complejidades que le afectan directamente al aprendiz, de ahí a que sea una de las principales preocupaciones de los docentes del lenguaje, e incluso de otras áreas, como las ciencias sociales, o la biología e incluso la historia. El mal manejo de la lectura es un problema vertebral: puede decirse sencillamente que uno de los problemas de la escritura es la falta de lecturas, tal y como lo diría Álvarez, en la medida en que el escritor, en este caso el educando adolecería de prerrequisitos de lectura, hecho que imposibilitaría la escritura, pero el problema de la falla en la lectura, en multidimensional, y muchos autores han reflexionado al respecto, con el propósito de generar un acercamiento a la problemática y ofrecer una alternativa viable, que contribuya significativamente a la adquisición de destrezas y competencias que permitan desarrollar ejercicios de lectura más precisos y complejos. Esta proliferación de información hace que la lectura sea un proceso inacabado, vulnerable a la interacción e intervención constante en el aula.

En este orden de ideas, según Sánchez las falencias presentes en ciertos educandos se debe a que ellos (los educandos) se concentran solo en decodificar el texto mas no en comprenderlo e interpretarlo, realizando así una lectura superficial que no llega a la interiorización. Tomando como punto de partida los trabajos de Smith (1978) propone que la lectura es un proceso interactivo en el que dicho lector, debe partir no de la decodificación sino de la interiorización y de esta forma, poner en juego conocimientos previos, relacionándolos con los nuevos contenidos. Lo que daría como resultado una lectura reflexiva, una lectura encaminada a un propósito educativo: a la generación de un tipo de individuo más crítico y reflexivo de su propio entorno, de su propio mundo.

Ahora bien, retomando un poco la idea sobre la preocupación y búsqueda de las distintas formas de abordar la lectura y la escritura en el aula, dentro de esa intervención constante a la que se someten por su dificultad 
implícita, Juan E Jiménez e Isabel O’Shanahan (2008) interrelacionan los planteamientos fundamentales en la enseñanza de la lectura y la analizan a partir de la revisión de una serie de investigaciones situadas desde distintos enfoques, buscando entre ellas un punto común desde el cual, sea posible aplicar las teorías de la enseñanza de los anteriores elementos en el individuo.

Estos autores, construyen sus aportes desde un enfoque sociocultural y constructivista, desde el que señalan la "importancia de las interacciones sociales en los procesos cognitivos de aprendizaje" (Juan E Jiménez, 2008), haciendo hincapié en la idea que el aprendizaje de la lectura y la escritura se inicia en espacios no formales; en el contexto familiar, resalta que los niños poseen un conocimiento previo a la lectura, por lo que es de vital importancia generar un acompañamiento que trascienda los muros de la escuela. Estos autores proponen una lectura, tal y como se ha señalado en líneas anteriores, con la capacidad de articular distintos factores del exterior, a lo que ellos nominan "lectura dialógica", una lectura centrada en la creación de sentido a partir de las distintas posibles relaciones que puedan nacer de ella; se articula el proceso contextualizador de una práctica docente encaminada a rescatar el valor práctico de lo que se lee para la vida. Tal vez nunca antes, a partir de la Edad Media hubo tanta preocupación por el individuo, y la lectura y sobre todo por la lectura de su mundo, por ello, ante el intento de resarcir esa brecha educativa que duró casi cuatro siglos, los maestros y pedagogos proponen a la lectura como un punto de reflexion constante en el aula, ya que después de tantas propuestas aún se siguen ofreciendo alternativas cada vez mas novedosas que ayudan a subsanar los problemas lectoescriturales de los estudiantes.

En ente orden de ideas, Yvonne S. Freeman (s.f.) bajo una rigurosa revisión teórica titulada "Métodos de lectura en español: ireflejan nuestro conocimiento actual del proceso de lectura?" presenta nueve metodos de enseñanza de la lectura que son: el método alfabético, el onomatopéyico, el fónico o fonético, el silábico, el de palabras generadoras, el método global, el método global puro, el ideovisual y el ecléctico e integral. Todos como producto de una reflexión propia de la práctica de enseñanza en el aula; plantea que "los expertos en la enseñanza de la lectura están preocupados porque a pesar de las investigaciones y teorías sobre el proceso de lectura, la aplicación de investigaciones recientes y de las teorías resultantes no son evidentes en el aula"(Freeman, s.f.) esto pone en relieve la precupacion latente sobre los procesos de escritura en el aula, haciéndolos un elemento de intervención continua, sobre el que no se deja de reflexionar. 
A raíz de esto, Miscues (Freeman, s.f.) realizó una seríe de estudios donde concluye que el proceso de lectura incluye más que el reconocimiento de palabras, pues el lector no solo tiene conociminto grafofónico sino también los conocimientos previos adquiridos desde la experiencia y sostiene que los docentes deben mostrar su conocimiento del proceso de lectura para que tomen posición sobre este y evalúen los textos que se utilizarán en clases y con ello hacer de la lectura un proceso mucho mas dialógico y menos intrumentalizado.

\section{Experiencia y "prácticas sociales"}

(Doria, 2000) en su texto Aproximación al ensayo literario en la escuela, enfoques metodológicos, afirma que no existen prácticas discursivas sin práctica social. Desde esta mirada un tanto sociológica, existen autores que reconocen el componente social, además de la experiencia como elemento indispensable en el ejercicio mismo de la escritura y por ende la lectura; definen esa relacion inherente entre la escriura y las prácticas sociales, puesto que nos encontramos dentro de un entorno que debe ser leído (...) y la pretención sería que el más modesto programa de la modernidad tenga que dar vigencia a una sociedad que ejerce, respeta y promueve el derecho a leer el mundo (Larrosa, y otros, 2008).

En el mismo sentido Cassany (1999) en el artículo "Construír la escritura" establece que la práctica escritural es producto de las interacciones sociales pero que va a estar determinada por las herencias socioculturales que posea el colectivo humano, además de constituirse como un acto complejo que se define estructuralmente de acuerdo a una serie de patrones y estímulos que se van asimilando con el tiempo. En lo concerniente al aula, el autor resalta la necesidad de los ejercicios de socialización y por ello reconoce la relación entre la escritura y los actos comunicativos.

En este sentido, la escritura esta visionada desde Cassany como una práctica social, puesto que va a estar mediada por las influencias culturales de la comunidad a la cual pertenece el individuo.

Sumado a lo anterior Cassany (2002) en otro de sus articulos titulado, "construccion de la escritura" menciona la necesidad de la constitución del pensamiento crítico como una herramienta de lucha ante las situaciones poco democráticas que se presentan actualmente. Esto resalta nuevamente la búsqueda en cuanto al propósito social y práctico de la lectura y la escritura, el autor reconoce la demanda necesaria de seres autónomo, propositivos 
y comprometidos socialmente, en sociedades democráticas propositivas y comprometidas socialmente. En el texto de este autor se reconocen tres tipos de procesos de lectura: leer las líneas (literalmente), leer entre las líneas, (la capacidad de inferencia del lector con relación al texto) y leer detrás de las líneas (juicio crítico que emite el lector con relación al texto leído), siendo este último el tipo de lectura al que debería llegar el ser humano. Este tipo de método se ubica dentro de un enfoque comunicativo, en la medida en que busca ir más allá de la literalidad del texto, enfocándose en las interpretaciones que pueden surgir del mismo; en este paradigma se tiene en cuenta no solo el acto de leer en sí mismo, sino su función comunicativa (en relación con el entorno).

De este modo, en el anterior artículo se maneja una tendencia crítica de lectura, una lectura minuciosa, reflexiva y analítica que permite el reconocimiento de la realidad a la cual pertenece el individuo y a través de ello tomar posición frente a las problemáticas sociales. La lectura crítica tiene como finalidad crear seres con posiciones sociales definidas mediante argumentos "objetivos".

De igual manera, Larrosa (2003) en su texto "La experiencia de la lectura. Estudios sobre literatura y formación" plantea una posición transgresora, de acuerdo a la pedagogía imperante hasta el siglo pasado y cuyos sustratos siguen vigentes. Este autor propone a partir de su evidente influjo por la filosofía de Gadammer y su concepto de experiencia trabajado en el texto "verdad y método" la lectura como un contacto directo entre las subjetividades; un encuentro con el otro. Señala, como punto de partida, que la lectura debe ser un proceso transparente, de abertura, de entrega, en el que el lector se permite a sí mismo ser atravesado por la lectura para conectarse con su "YO", punto en que la lectura como un hecho "que no pasa, sino que nos pasa" se convierte en experiencia y esta experiencia se transforma en formación en la medida en que el lector sea capaz de estar atento, de escuchar y no reducirlo todo a su mirada.

En esta misma línea, Álvaro Marín Marín (s.f.), en su reflexión titulada "Propuesta de modelo didáctico para el fomento de la lectura recreativa en la secundaria" plantea desde un punto de vista hermenéutico interpretativo gadammeriano, que la enseñanza de la lectura debe estar unida a los intereses de la escuela y de los mismos profesores, pues la escuela como espacio de acción para la enseñanza tiende a coartar las prácticas docentes con sus mallas curriculares. No obstante, el papel del profesor también debe ser activo, no 
solo para juzgar y accionar en la escuela misma sino para reconocer sus propias falencias y proponer una lectura contextualizada que despierte el interés de los educandos y así mismo, mejore la experiencia de la lectura en la medida en que esta sea recreativa. En este texto no solo se evalúan las deficiencias cognitivas de los estudiantes sino que vuelca la atención en la importancia de un verdadero gusto de lectura por parte del docente que es el que en última instancia, generará el contacto ya sea positivo o negativo en el aprendiz, por lo que se requiere un verdadero compromiso intelectual. Una de sus premisas fundamentales, es que:

cuando los jóvenes superan la trampa del lenguaje limitado y se atreven a tomar la palabra, no sólo están tratando de salir de la marginación cultural con todas sus limitaciones, sino que también, inician la posibilidad de una ciudadanía activa y participativa, que tanta falta nos está haciendo en momentos de transición como los actuales. (Marín, 2012:04).

Lo que ya le atribuye a la lectura un carácter social unido a la posibilidad de encontrar y redescubrir a través de ella distintos caminos que se vinculan con la realidad. Desde esta postura, la experiencia personal, la ensoñación y la imaginación recobran vitalmente su valor más alto.

\section{La inclusión de las TIC'S como instrumento para las prácticas de lectura y escritura}

$\mathrm{P}$ or otro lado, los avances tecnológicos han traído por sí mismos mejoras en distintos sectores sociales, hecho que le ha dado a dichos avances vital relevancia, gracias a su carácter "práctico". No obstante, se han presentados algunos inconvenientes a la hora de introducir las herramientas tecnológicas, como recursos, al ámbito educativo por factores muy diversos, pero entre los más destacados se encuentra la falta de dominio de la herramienta.

La inclusión de las tecnologías a la sociedad ha traído distintos modos de acercamiento a los textos, por ello, también ha generado preocupación para los docentes. La lectura por si ya es un acto complejo, tal y como lo plantea Cassany, y si a ello se le agrega la imposibilidad actual de una lectura crítica del mundo tal y como se viene mencionando a lo largo de este documento, el proceso de enseñanza o de acompañamiento de el desarrollo de estos procesos (leer y escribir) se hace un poco más tortuoso y difuso. Por eso Lomas (2003) en su artículo "Leeer para entender y trnsformar el mundo", concibe 
la nueva era digital como un factor negativo, en la medida en que se hace una mala utilización de los recursos, lo que conlleva a una mala utilización de la información, y con ello a una posición frente a los problemas sociales en extremo pasiva. Con tal afirmación queda clara la intención del autor, y su visión sobre lo que es la lectura, y su función social. Al dotar a la lectura y a la escritura de axiomas que la enfocan con miras a un propósito social, queda más que claro el hecho de que estas dos actividades han sido el producto del prurito de la reflexión, encaminado a una sociedad letrada, la cual solo es posible desde espacios de intervención como el aula.

En este artículo se reconoce cómo funciona el lenguaje en su contexto social y cultural, por eso reconoce la importancia de la lectura y su comprensión para entender y transformar la visión de mundo que se posea y salir del estado "sumiso" de la que los medios masivos de comunicación provocan al no saber elegir y seleccionar la información adecuada.

Desde el manejo de las TICs, Juan E Jiménez, (2008) en su artículo "Enseñanza de la lectura: de la teoría y la investigación a la práctica educativa" plantea que el ordenador, como herramienta complementaria para la enseñanza de la lectura y escritura, por incluir diversos elementos visuales en esa unidad llamada texto, puede facilitar el aprendizaje, puesto que este instrumento sirve como un mediador, que no por reforzar la información con imágenes o gráficas debe estar aislado del acompañamiento docente.

Así como, Vásquez (2013) en su trabajo "Análisis y evaluación de la didáctica con base en lectura digital en el marco PISA" tiene como fin la adaptación en la escuela de la novedad digital y su búsqueda de metodologías para integrar los nuevos escenarios y herramientas virtuales en el proceso de enseñanzaaprendizaje, a raíz de la cercanía y la facilidad con la que acceden los jóvenes a dispositivos tecnológicos y la forma en que dichos artefactos acercan al estudiante a la lectura. El autor incita a la implementación de las TICs como una herramienta eficaz para promover espacios interactivos para el acto educativo. Se considera que las escuelas deben adaptarse a la innovación que ofrece la época actual, reconociendo la necesidad de que los docentes formen parte de esta nueva oleada de tecnología digital para que puedan ser capaces de reconocer su utilidad y crear conciencia de ellas, y a su vez, creen didácticas ciberlingüísticas que promuevan la creación de textos digitales.

En relación con lo anterior, Cánovas (2007) en su artículo "La lectura y las nuevas tecnologías" reconoce las TIC's como un camino alternativo para el 
aprendizaje que no debe descuidarse, pues en "la cultura de la información" los constantes avances han empezado a adentrarse en las aulas y las bibliotecas, hecho que ha alterado sustancialmente la actividad tradicional de docente y del bibliotecario los cuales, necesitan mayor inclusión y acercamiento de estos a las herramientas digitales, ya que actualmente los jóvenes están frente a estos recursos frecuentemente y desde un punto de vista práctico, esto debe aprovecharse para acercarlos a la lectura y al conocimiento a través de ella. Por tal motivo, se hace perentoria la necesidad de reeducarse como lectores polivalentes y multimediales, capaces de reconocer el soporte medial como una herramienta cotidiana de la comunicación que contribuye al desarrollo de la lectura como práctica cotidiana.

\section{Conclusiones}

- Las reflexiones sobre la lectura y la escritura, de acuerdo a la disparidad de los casos en la escuela, ha generado una preocupación sobre la práctica misma, hecho que ha ocasionado un gran cúmulo de posturas que ofrecen la posibilidad de repensar los métodos de enseñanza.

- A partir del análisis de las diferentes posturas teóricas que se hallan en los documentos abordados en este trabajo, se asume como tendencia predominante el social constructivista, orientado a explotar la experiencia que posee el individuo, reconociendo la importancia de los preconceptos para el desarrollo de nuevos aprendizajes.

- Las investigaciones están direccionadas a la configuración, comprensión e integración de los nuevos conceptos como producto de las interacciones con el medio, con el objetivo de fomentar la argumentación y el desarrollo discursivo del individuo. Además, tiene como finalidad crear sujetos comunicativos, críticos-reflexivos que sean capaces de configurar su visión de mundo.

- Se busca que el maestro centre el interés en crear situaciones que le permitan al estudiante construir su propio discurso en el contexto social y cultural a partir de la creación de estrategias didácticas alejadas de la forma tradicional de enseñar.

- Las prácticas de enseñanza deben basarse en una evaluación constante que le permita tanto al estudiante como al maestro, reconocer falencias y potenciar las mejoras. 
- La lectura es vista desde una mirada semiótica, puesto que el proceso se constituye como un diálogo entre el texto, la sociedad y los presaberes del lector, fomentando en este sentido actos de pensamientos a través del proceso de lectura.

- Las TICS deben empezar a ser incluidas en la enseñanza de la lectura y escritura, debido a las transformaciones tecnológicas que modifican las relaciones humanas, mediante el cual las personas se involucran en nuevas formas de participación e interacción social y de este modo, pueden ser vistas como herramientas didácticas que intervienen en los procesos de enseñanza y de aprendizaje puesto que la gran cantidad de información que se puede obtener bajo dicho elemento, si es bien seleccionada, puede contribuir decisivamente en la constitución de la visión de mundo del individuo crítico y reflexivo.

\section{BIBLIOGRAFÍA}

Álvarez, C. (1993). Técnicas de lectura eficaz. Recuperado el 8 de marzo de 2015, de http://aufop. com/aufop/uploaded_files/articulos/1273155552.pdf

Sánchez, E. (1988). Aprender a leer y leer para aprender: Características del escolar con pobre capacidad de comprensión. Recuperado el 8 de marzo de 2015, de Google: dialnet.unirioja.es/ descarga/articulo/48315.pdf

Cassany. (1999). Construir la escritura. Recuperado el 8 de marzo de 2015, de http:// www.ucv.ve/fileadmin/user_upload/sadpro/Documentos/docencia_vol2_n2_2001/12_ rese\%C3\%B1a_5Daniel_Cassany.pdf

Cassany. (s.f.). Construcción de la escritura. Recuperado el 8 de marzo de 2015, de http:// wWw.ucv.ve/fileadmin/user_upload/sadpro/Documentos/docencia_vol2_n2_2001/12_ rese\%C3\%B1a_5Daniel_Cassany.pdf

Cassany, D. (2002). Aproximaciones a la lectura crítica: teoría, ejemplos y reflexiones. Tarbiya, 113-132.

Doria, R. (2000). Aproximación al ensayo literario en la escuela, enfoques metodológicos. Montería: Ediciones Paloma.

Doria, R., \& Pacheco, M. (2012). Dinámicas de investigación-acción participativa en la generación de experiencias de lectura y escritura en la escuela. Montería: Universidad de Córdoba.

Freeman, Y. S. (s.f.). Recuperado el 5 de 5 de 2015, de Métodos de lectura en español: ¿̇reflejan nuestro conocimiento actual del proceso de lectura?: http://www.lecturayvida.fahce.unlp.edu. ar/numeros/a9n3/09_03_Freeman.pdf 
Lomas, C. (2003). Leeer para entender y trnsformar el mundo. Recuperado el 8 de marzo de 2015, de http://www.uv.mx/personal/lenunez/files/2013/06/LR02_LeerEscribirEntenderMundo.pdf

Reyes, Y. (2005). Lectura en primera ifancia Recuperado el 8 de marzo de 2015, de Google: http:// www.cerlalc.org/redplanes/secciones/biblioteca/reyes_lectura_primera_infancia.pdf

O'shanahan, J. (2008). Enseñanza de la lectura: de la teoría y la investigación a la práctica educativa. Recuperado 8 de marzo de 2015, de http://www.rieoei.org/deloslectores/2362JimenezV2.pdf

Jiménez, Juan E. I. O. (2008). Enseñanza de la lectura: de la teoría y la investigación a la práctica educativa. Revista Iberoamericana de Educación, 5/25.

Larrosa, J. (s.f.). La experiencia de la lectura. Recuperado el 21 de 04 de 2015, de http://infocuib. laborales.unam.mx/ mt12s01k/archivos/data/1/8.pdf

Larrosa, J.; Fernández, L.; Delgado, H.; Huck, F. V.; Castrillón, S.; Rodríguez, A. \& Otros. (2008). Leyendo en Babel, lectura educación y ciudad. Cali: Icesi.

Marín, Á. (s.f.). Recuperado el 1 de 5 de 2015, De propuesta de modelo didáctico para el fomento a la lectura recreativa en la secundaria: http://www.umass.edu/complit/aclanet/A_Marin.html

Vásquez, E. (2013). Análisis y evaluación de la didáctica con base en lectura digital en el marco PISA: el caso de España. Recuperado el 8 de marzo de 2015, de http://www.rinace.net/riee/numeros/ vol6-num1/art04.html 